\title{
Comparação de métodos de detecção de fungos em sementes de cebola
}

\author{
Comparison of methods for the detection of \\ fungi in onion seeds
}

Juliana Ferreira de Moura ${ }^{1}$, Regina Ceres Torres da Rosa ${ }^{1 *}$,

Ana Patrícia dos Santos Gonçalves ${ }^{1}$, Luciana Melo Sartori Gurgel' ${ }^{1}$, Tereza Cristina de Assis ${ }^{1}$,

Domingos Eduardo Guimarães Tavares de Andrade', Venézio Felipe dos Santos ${ }^{1}$

'Laboratório de Patologia de

Sementes, Instituto Agronômico de Pernambuco (IPA),

Av. General San Martin, 1371

Bongi, CEP $50761-000$

Recife, PE, Brasil

*autor correspondente

凶 reginactrosa@gmail.com
RESUMO: Em experimentos conduzidos em laboratório, diferentes substratos [papel-filtro, batata-dextrose-ágar (BDA), V-8-ágar, meio ágar-água (AA)] foram comparados visando selecionar o mais sensível para detecção de fungos em sementes de cinco cultivares de cebola (Roxa IPA-03, Composto IPA-06, Franciscana IPA-10, Vale ouro IPA-11 e Brisa IPA-12). Os substratos foram testados sem e com congelamento $\left(-20^{\circ} \mathrm{C}\right.$, durante 24 horas) das sementes. Foram identificados os seguintes fungos: Aspergillus sp., A. niger, A. flavus, Penicillium sp., P. digitatum, Colletotrichum gloeosporioides f. sp. cepae, Fusarium sp., F. oxysporum, F. graminearum, F. equiseti, Chaetomium globosum, Curvularia lunata, Curvularia sp., Papilaria sp. e Rhizopus sp. Aspergillus niger foi o mais frequentemente detectado em todos os tratamentos estudados. Sob congelamento, os meios mais sensíveis para detecção de A. niger e A. flavus foram BDA e V8, respectivamente. As cultivares IPA-11 e IPA-6 obtiveram altas percentagens de A. niger.

PALAVRAS-CHAVE: Allium cepa, patologia de sementes, método blotter, meios de cultura.
ABSTRACT: This work was carried out to compare and evaluate different cultivars (Roxa IPA-03, Composto IPA-06, Franciscana IPA-10, Vale ouro IPA-11 e Brisa IPA-12) and culture media used in the laboratory, with the purpose of selecting the more sensitive detection of onion seed fungi. In lab experiments, various methods for seed blotter testing; PDA medium; V-8 medium and water-agar medium were compared regarding the detection of pathogens in onion seeds. The following fungi were identified: Aspergillus sp., A. niger, A. flavus, Penicillium sp., P. digitatum, Colletotrichum gloesporioides $f$. sp. cepae, Fusarium sp., F. oxysporum, F. graminearum, F. equiseti, Chaetomium globosum, Curvularia lunata, Curvularia sp., Papilaria sp. and Rhizopus sp. Aspergillus niger was the most frequently detected fungus in all treatments. Results showed that under seed freezing $\left(-20^{\circ} \mathrm{C}\right.$ for 24 hours), the selective media were more sensitive in the detection of $A$. niger and A. flavus, followed by BDA and V8, respectively. The cultivars IPA-11 and IPA-6 presented large percentages of A. niger.

KEYWORDS: Allium cepa, seed pathology. blotter test. culture media.

\section{Introdução}

As sementes são eficientes meios de disseminação e transmissão de patógenos e, frequentemente os introduzem em áreas isentas (NEERGAARD, 1983). Com o advento do livre comércio, muitos países estão redefinindo suas exigências fitossanitárias com o objetivo de prevenir a introdução de patógenos devastadores em seu país. Independentemente da metodologia de detecção, a especificidade, a sensibilidade, a confiabilidade, a eficiência do ensaio e uma compreensão de tolerância de patógenos em um lote de sementes precisam ser 
considerados antes de uma técnica ser aceita como um teste clínico sanitário de semente. Quando aceitáveis, os testes sanitários são ferramentas úteis para a gestão de risco de doença e rotineiramente utilizados na avaliação da qualidade das sementes (MADDOX, 1998). Sendo assim, há de se considerar, também, a necessidade de estabelecimento de padrões sanitários de sementes no Brasil.

$\mathrm{Na}$ literatura, têm sido publicados diversos trabalhos relacionados à detecção de fungos em sementes de diferentes culturas e métodos, sendo o teste com papel de filtro o mais amplamente conhecido e utilizado, embora a incidência de contaminantes, como fungos e bactérias, que crescem rapidamente neste substrato, possa impedir a frutificação dos fungos-alvo, dificultando a sua identificação e quantificação, sobretudo os de crescimento lento (REIS et al., 1999; ALMEIDA; REIS, 2009).

Outro método de detecção de fungos, com possibilidade de uso em análises rotineiras de patógenos de sementes, são os meios seletivos e semisseletivos (REIS et al., 1999). Entre os protocolos indicados pelo Ministério da Agricultura, Pecuária e Abastecimento-MAPA (BRASIL, 2009) estão a inspeção visual das amostras de sementes, exame da suspensão de lavagem, meio ágar sólido (BDA ou MEA), método do papel de filtro e específicos para determinadas espécies fúngicas. Para Lucca Filho (1987), os meios de cultura devem ser utilizados quando outros não ofereçam condições adequadas para crescimento vegetativo, esporulação e detecção de fungos que produzam colônias características. Os meios de cultura favorecem a germinação das sementes, podendo prejudicar a identificação dos fungos a elas associados (COUTINHO et al., 2001). O meio ágar suco de tomate, mais $6 \%$ de cloreto de sódio, é recomendado para detecção de fungos de armazenamento, como o gênero Aspergillus, que se desenvolve em alta concentração osmótica. Esse meio, também, não favorece a germinação das sementes, facilitando a identificação de fungos ao examinar as sementes inteiras (NEERGAARD, 1983).

Atualmente, poucos trabalhos relacionados a métodos de detecção de fungos em sementes de cebola têm sido encontrados. Assim, o presente trabalho teve como objetivo comparar e avaliar diferentes métodos para a detecção de fungos em 5 cultivares de cebola.

\section{Material e Métodos}

A pesquisa foi conduzida no Laboratório de Patologia de Sementes do Instituto Agronômico de Pernambuco-IPA. Utilizaram-se sementes de 5 cultivares de cebola (Roxa IPA-03, Composto IPA-06, Franciscana IPA-10, Vale ouro IPA-11 e Brisa IPA-12).

Os tratamentos foram constituídos de diferentes substratos BDA (extrato de $200 \mathrm{~g}$ de batata $+20 \mathrm{~g}$ de dextrose + 17 de ágar/L), ágar-água - AA, V8 (meio com $200 \mathrm{~mL}$ suco $+2,0 \mathrm{~g}$ de $\mathrm{CaCO}_{3} / \mathrm{L}$ ) e papel de filtro e dois métodos de incubação, sem e com congelamento.

\section{Método de incubação de sementes sem congelamento}

As sementes foram desinfestadas com solução de $\mathrm{NaClO} 2 \%$ por 3 minutos, água destilada esterilizada (ADE) na proporção de 1:3 (v:v) por 1 minuto e lavagem por duas vezes em ADE. Em seguida, 20 sementes de cada cultivar foram colocadas em placas de Petri, contendo os substratos citados acima e em condições assépticas. Logo após, as sementes foram incubadas em luz fluorescente durante 7 dias, à temperatura de $28 \pm 2{ }^{\circ} \mathrm{C}$, sob alternância luminosa (12 horas claro/12 horas escuro), em câmara de crescimento.

\section{Método de incubação de sementes com congelamento}

Depois da desinfestação das sementes, realizada da mesma maneira descrita no item anterior, as sementes foram mantidas em câmara de crescimento à temperatura de $25 \pm 2{ }^{\circ} \mathrm{C}$, sob alternância luminosa (12 horas claro/12 horas escuro), durante 24 horas. Depois desse período, foram congeladas a $-20 \pm 2{ }^{\circ} \mathrm{C}$, pelo mesmo tempo, sendo, em seguida, submetidas aos diferentes substratos e incubadas nas condições já referidas anteriormente.

O teste de patogenicidade foi realizado com os fungos detectados no levantamento que apresentaram maior frequência e importância, para tanto foram cultivados em meio BDA por sete dias. Em seguida, as sementes foram imersas, por 30 minutos, em uma suspensão de $10^{6}$ conídios $/ \mathrm{mL}$ de cada fungo, secas à temperatura ambiente e submetidas ao teste do papel de filtro. Das sementes ou plântulas que desenvolveram sintomas, foram retirados fragmentos e realizado o isolamento. A avaliação da patogenicidade foi feita verificando-se os sintomas causados pelos fungos inoculados com o auxílio de microscópio óptico.

As avaliações quantitativa e qualitativa dos fungos associados às sementes foram realizadas após 7 dias de incubação, examinando-se, individualmente, as sementes ao microscópio estereoscópico e microscópio óptico. Os gêneros dos fungos foram identificados com base em suas características morfológicas e as espécies, com o auxílio de literatura específica. O delineamento estatístico foi o inteiramente casualizado, com 10 repetições/tratamento. Os dados foram submetidos à análise de variância e teste de Tukey, ambos a $5 \%$ e transformados em arco-seno raiz $((x+1) / 100)$.

\section{Resultados e Discussão}

No geral, foram identificados nas sementes de cebola os seguintes fungos: Aspergillus sp., A. niger, A. flavus, Penicillium sp., P. digitatum, Colletotrichum gloeosporioides f. sp. cepae, Fusarium sp., F. oxysporum, F. graminearum, F. equiseti, Chaetomium globosum, Curvularia lunata, Curvularia sp., Papilaria sp. e Rhizopus sp. (Figura 1). No teste de patogenicidade realizado com os fungos $A$. niger, A. flavus, Penicillium sp., Curvularia lunata, Fusarium sp. e Chaetomium globosum, apenas o último não foi patogênico. Nos meios BDA e V8, observou-se ampla gama de espécies fúngicas nas sementes e, para alguns deles, alta incidência, 
quando comparados com os demais substratos (Tabela 1). A Curvularia lunata apresentou maior incidência sobre o meio AA, independente da cultivar e temperatura utilizada. Aspergillus flavus e C. gloeosporioides f. sp. cepae foram detectados nas cultivares IPA-3 e IPA-10, respectivamente. A presença do gênero Colletotrichum, mesmo com uma incidência menor nas cultivares estudadas, demonstra a necessidade do monitoramento e controle da qualidade sanitária na produção de sementes, devido à importância desse patógeno para a cultura da cebola e por constituir uma das formas de transmissão e disseminação da antracnose, ou mal de sete voltas, nos campos de produção (MASSOLA JUNIOR; JESUS JUNIOR; KIMATI, 2005; HILL, 2008).

Observou-se a associação de diferentes espécies de Fusarium às sementes das cultivares de cebola, contudo, a importância patológica e epidemiológica desta associação tem sido pouco estudada (BOFF et al., 1995). Segundo (MANNERUCCI et al., 1987; KLOKOÅAR-ŠMIT et al., 2008), as espécies mais frequentemente associadas à podridão de cebola no campo e armazenamento são $F$. oxysporium f.sp. cepa, $F$. solani e $F$. proliferatum, causando, respectivamente, a podridão basal, retardando o crescimento inicial e produção de importantes micotoxinas.

Em todos os tratamentos estudados, independente do meio utilizado e das mesmas terem sido congeladas ou não, constatou-se maior incidência de $A$. niger que diferiu das demais espécies identificadas (Tabela 2). Estes resultados estão de acordo com El-Nagerabi e Abdalla (2004) que, utilizando os substratos BDA e papel de filtro, identificaram 19 gêneros, destacando-se o Aspergillus, com, aproximadamente, 42,1\% das colônias fúngicas encontradas. Entre as espécies deste gênero, mais de $19 \%$ das colônias detectadas eram de $A$. niger. Ainda de acordo com esses autores, o meio BDA apresentou maior quantidade e diversidade de fungos do que no papel de filtro.

Os resultados demonstram que, no tratamento com congelamento $\left(-20{ }^{\circ} \mathrm{C}\right.$ por 24 horas), os meios mais sensíveis para detecção de A. niger e A. flavus, foram BDA e V8, respectivamente (Figura 2). Em estudo com sementes Pterogyne nitens, utilizando-se diferentes métodos de detecção,
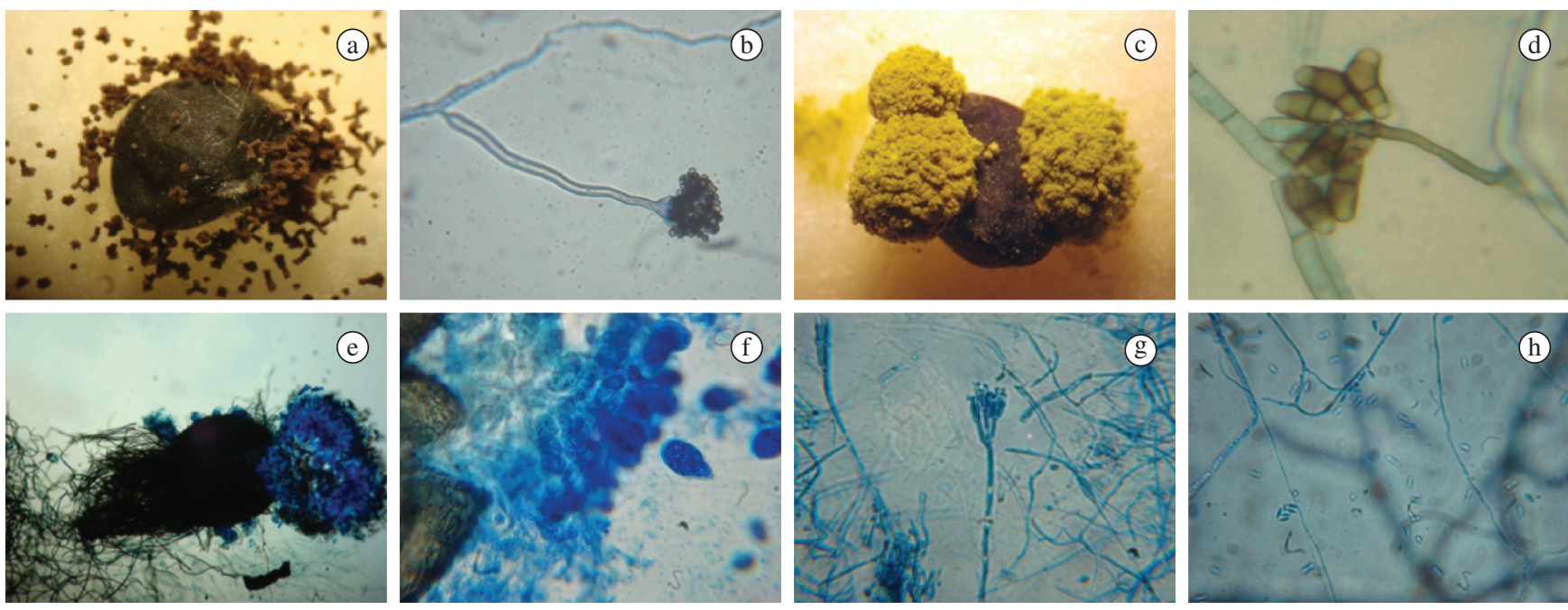

Figura 1. Alguns fungos detectados em sementes de cebola, a) e b) Aspergillus niger; c) Aspergillus flavus; d) Curvularia lunata; e) e f) Chaetomum globosum; g) Penicillium sp.; h) Fusarium sp.

Tabela 1. Incidência média dos fungos detectados em diferentes substratos, independente da cultivar e do congelamento ou não das sementes de cebola.

\begin{tabular}{|c|c|c|c|c|c|c|c|c|c|c|c|c|c|c|c|}
\hline \multirow{2}{*}{ Substrato } & \multicolumn{15}{|c|}{ Incidência de fungos (\%) } \\
\hline & $\mathbf{A}$ & An & Af & $\mathbf{F}$ & $\mathbf{F e}$ & $\mathbf{F g}$ & Fo & $\mathbf{P}$ & Pd & $\mathrm{Cg}$ & $\mathbf{C}$ & Cl & Co & $\mathbf{P a}$ & $\mathbf{R}$ \\
\hline BDA & $0,50 \mathrm{a}$ & 52,40 a & $0,40 \mathrm{ab}$ & $0,10 \mathrm{a}$ & $0,00 \mathrm{a}$ & $0,05 \mathrm{a}$ & $0,00 \mathrm{a}$ & $0,05 \mathrm{ab}$ & $0,00 \mathrm{a}$ & $0,05 \mathrm{a}$ & $0,20 \mathrm{a}$ & $0,00 \mathrm{~b}$ & $0,10 \mathrm{a}$ & $0,00 \mathrm{a}$ & $0,45 \mathrm{ab}$ \\
\hline V8 & $0,00 \mathrm{a}$ & $35,20 \mathrm{~b}$ & 0,85 a & $0,10 \mathrm{a}$ & $0,05 \mathrm{a}$ & $0,00 \mathrm{a}$ & $0,05 \mathrm{a}$ & $0,10 \mathrm{ab}$ & $0,00 \mathrm{a}$ & $0,00 \mathrm{a}$ & $0,05 \mathrm{a}$ & $0,00 \mathrm{~b}$ & $0,20 \mathrm{a}$ & $0,05 \mathrm{a}$ & $0,25 \mathrm{~b}$ \\
\hline AA & $0,00 \mathrm{a}$ & $24,05 \mathrm{c}$ & $0,10 \mathrm{a}$ & $0,00 \mathrm{a}$ & $0,05 \mathrm{a}$ & $0,00 \mathrm{a}$ & $0,00 \mathrm{a}$ & $0,00 \mathrm{~b}$ & $0,05 \mathrm{a}$ & $0,00 \mathrm{a}$ & $0,10 \mathrm{a}$ & $0,20 \mathrm{a}$ & $0,00 \mathrm{a}$ & $0,00 \mathrm{a}$ & $0,00 \mathrm{~b}$ \\
\hline Blotter test & $0,55 \mathrm{a}$ & $3,05 \mathrm{~d}$ & 0,10 a & $0,00 \mathrm{a}$ & $0,00 \mathrm{a}$ & $0,00 \mathrm{a}$ & $0,00 \mathrm{a}$ & $0,25 \mathrm{a}$ & $0,15 \mathrm{a}$ & $0,00 \mathrm{a}$ & $0,00 \mathrm{a}$ & $0,00 \mathrm{~b}$ & $0,00 \mathrm{a}$ & $0,00 \mathrm{a}$ & $1,05 \mathrm{a}$ \\
\hline CV (\%) & 40,51 & 24,39 & 35,66 & 13,89 & 10,05 & 6,94 & 6,94 & 19,41 & 14,78 & 6,94 & 12,88 & 13,02 & 18,37 & 6,94 & 38,83 \\
\hline
\end{tabular}

$\mathrm{A}=$ Aspergillus spp.; $\mathrm{An}=$ Aspergillus niger $; \mathrm{Af}=$ Aspergillusflavus $; \mathrm{F}=$ Fusarium $\mathrm{spp} . ; \mathrm{Fe}=$ Fusarium equisete $; \mathrm{Fg}=$ Fusarium graminearum $; \mathrm{Fo}=$ Fusarium oxyporium $\mathrm{P}=$ Penicillium spp.; $\mathrm{Pg}=$ Penicillium digitatum $\mathrm{C}=$ Curvularia spp.; $\mathrm{Cl}=$ Curvularia lunata $; \mathrm{Co}=$ Colletotrichum gloesporioides $\mathrm{f} . \mathrm{sp}$. cepae. $\mathrm{Pa}=$ Papilaria $;$ $\mathrm{R}=$ Rhizopus spp. Médias seguidas de mesma letra minúscula na coluna são estatisticamente iguais, de acordo com o teste de Tukey $(\mathrm{p} \leq 0,05)$. Dados transformados para Arco-seno raiz $((\mathrm{x}+1) / 100)$. 

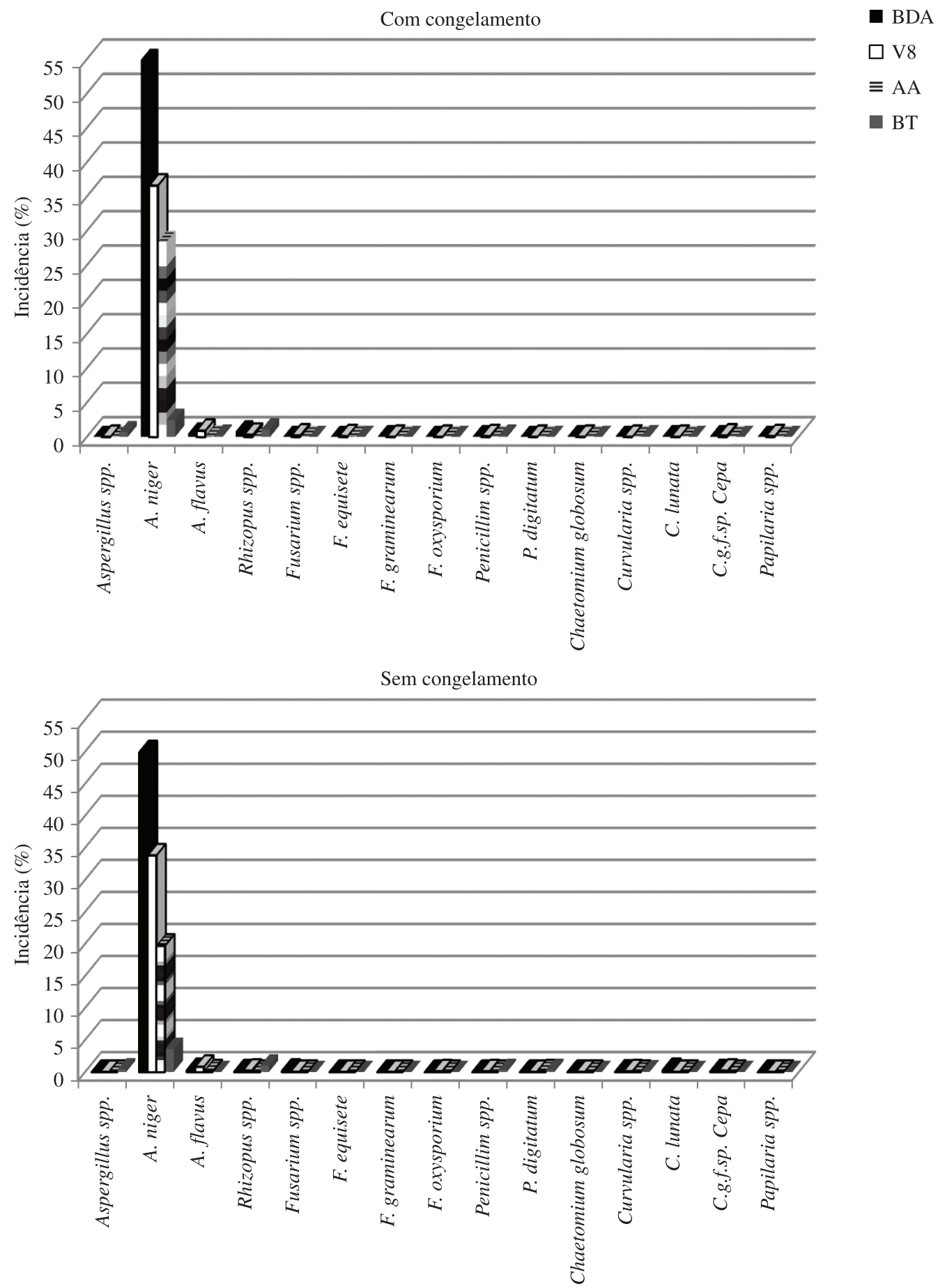

Fungos

Figura 2. Incidência média dos fungos em sementes de diferentes cultivares de cebola, com ou sem congelamento. BDA = batata-ágar-dextrose; AA = ágar-água; $\mathrm{V} 8=$ suco V8- $\mathrm{CaCO}_{3} ; \mathrm{BT}=$ método do blotter test .

verificou-se que, no papel de filtro com congelamento, houve redução na incidência dos fungos dos gêneros Aspergillus, Penicillium e Cladosporium (NASCIMENTO et al., 2006).

$\mathrm{Na}$ incidência média de $A$. niger, foi observada uma interação significativa entre cultivar, substratos e congelamento, observando-se maior incidência nas cultivares IPA-11 e IPA-6 (Tabela 3). Também se constatou no meio BDA, com ou sem congelamento, incidência de A. niger de 49,9\% e 54,9\%, respectivamente, que diferiu significativamente dos demais meios. A presença de Aspergillus em alta porcentagem reduz a viabilidade das sementes, por causar a perda da capacidade germinativa com a morte do embrião (MENTEN, 1995). De acordo com Lorbeer, Ransom e Tuffley (2000), A. niger pode ser capaz de infectar sementes de cebola através das flores do 
Tabela 2. Incidência média de fungos em sementes de diferentes cultivares de cebola independente do substrato e congelamento empregados.

\begin{tabular}{|c|c|c|c|c|c|}
\hline \multirow{2}{*}{ Fungo } & \multicolumn{5}{|c|}{ Cultivar (\%) } \\
\hline & IPA-3 & IPA-6 & IPA-10 & IPA-11 & IPA-12 \\
\hline Aspergillus spp. & $0,63 \mathrm{a}$ & $0,38 \mathrm{a}$ & $0,13 \mathrm{a}$ & $0,19 \mathrm{a}$ & $0,00 \mathrm{a}$ \\
\hline A. niger & $23,38 \mathrm{~b}$ & 39,56 a & $16,44 \mathrm{c}$ & $42,63 \mathrm{a}$ & $21,4 \mathrm{~b}$ \\
\hline A. flavus & $1,06 \mathrm{~b}$ & $0,38 \mathrm{~b}$ & $0,19 b$ & $0,19 b$ & $0,00 \mathrm{~b}$ \\
\hline Fusarium spp. & $0,00 \mathrm{a}$ & $0,00 \mathrm{a}$ & $0,19 \mathrm{a}$ & $0,00 \mathrm{a}$ & $0,06 \mathrm{a}$ \\
\hline F. equisete & $0,06 \mathrm{a}$ & $0,00 \mathrm{a}$ & $0,06 \mathrm{a}$ & $0,00 \mathrm{a}$ & $0,00 \mathrm{a}$ \\
\hline F. graminearum & $0,00 \mathrm{a}$ & $0,00 \mathrm{a}$ & $0,06 \mathrm{a}$ & $0,00 \mathrm{a}$ & $0,00 \mathrm{a}$ \\
\hline F. oxysporium & $0,00 \mathrm{a}$ & $0,00 \mathrm{a}$ & $0,06 \mathrm{a}$ & $0,00 \mathrm{a}$ & $0,00 \mathrm{a}$ \\
\hline Penicillium spp. & $0,00 \mathrm{a}$ & $0,19 \mathrm{a}$ & $0,19 \mathrm{a}$ & $0,13 \mathrm{a}$ & $0,00 \mathrm{a}$ \\
\hline P. digitatum & $0,00 \mathrm{a}$ & $0,00 \mathrm{a}$ & $0,00 \mathrm{a}$ & $0,00 \mathrm{a}$ & $0,25 \mathrm{a}$ \\
\hline Chaetomium globosum & 0,06 a & $0,00 \mathrm{a}$ & $0,00 \mathrm{a}$ & $0,00 \mathrm{a}$ & $0,00 \mathrm{a}$ \\
\hline Curvularia spp. & 0,06 a & $0,00 \mathrm{a}$ & $0,15 \mathrm{a}$ & $0,00 \mathrm{a}$ & $0,00 \mathrm{a}$ \\
\hline C. lunata & $0,00 \mathrm{a}$ & $0,00 \mathrm{a}$ & $0,19 \mathrm{a}$ & $0,00 \mathrm{a}$ & $0,06 \mathrm{a}$ \\
\hline Colletotrichum gloesporioides f. sp. cepae & $0,00 \mathrm{~b}$ & $0,00 \mathrm{~b}$ & $0,31 \mathrm{a}$ & $0,06 a b$ & $0,00 \mathrm{~b}$ \\
\hline Papilaria spp. & $0,00 \mathrm{a}$ & $0,00 \mathrm{a}$ & $0,00 \mathrm{a}$ & $0,00 \mathrm{a}$ & $0,25 \mathrm{a}$ \\
\hline Rhizopus spp. & $0,38 \mathrm{ab}$ & $1,00 \mathrm{a}$ & $0,00 \mathrm{~b}$ & $0,25 \mathrm{ab}$ & $0,56 a b$ \\
\hline $\mathrm{CV}(\%)$ & 35,86 & 27,53 & 30,33 & 26,85 & 29,57 \\
\hline
\end{tabular}

Médias seguidas de mesma letra minúscula na coluna são estatisticamente iguais, de acordo com o teste de Tukey ( $\mathrm{p} \leq 0,05)$. Dados transformados para arco-seno raiz $((x+1) / 100)$.

Tabela 3. Incidência média do Aspergillus niger em sementes de cultivares de cebola, com e sem congelamento, sob diferentes substratos.

\begin{tabular}{|c|c|c|c|c|c|c|c|c|c|}
\hline \multirow{3}{*}{ Cultivar } & \multicolumn{4}{|c|}{ Sem congelamento } & \multicolumn{4}{|c|}{ Com congelamento } & \multirow{3}{*}{$\begin{array}{l}\text { Média geral } \\
\text { Cultivares }\end{array}$} \\
\hline & \multicolumn{4}{|c|}{ Substratos } & \multicolumn{4}{|c|}{ Substratos } & \\
\hline & BT & $\mathbf{A A}$ & BDA & V8 & BT & AA & BDA & V8 & \\
\hline Roxa IPA 03 & $0.5 \mathrm{Bc}$ & $8,0 \mathrm{cdC}$ & $53,5 \mathrm{aA}$ & $36,5 \mathrm{aB}$ & $0.5 \mathrm{aC}$ & $17,5 \mathrm{bB}$ & $37,0 \mathrm{cA}$ & $33,5 \mathrm{bA}$ & $23,38 \mathrm{~b}$ \\
\hline Composto IPA 06 & $4.0 \mathrm{abC}$ & $30,5 \mathrm{abB}$ & $53,5 \mathrm{aA}$ & $49,5 \mathrm{aA}$ & $1.5 \mathrm{aC}$ & $43,5 \mathrm{aB}$ & $68,5 \mathrm{aA}$ & $65,5 \mathrm{aA}$ & 39,56 a \\
\hline Franciscana IPA 10 & $0.0 \mathrm{bC}$ & $1,0 \mathrm{dC}$ & $49,0 \mathrm{aA}$ & $18,0 \mathrm{bB}$ & $0.5 \mathrm{aB}$ & $1,0 \mathrm{cB}$ & $52,5 \mathrm{bA}$ & $9,5 \mathrm{cB}$ & $16,44 \mathrm{~b}$ \\
\hline Vale ouro IPA 11 & $13.5 \mathrm{aC}$ & $37,0 \mathrm{aB}$ & $63,0 \mathrm{aA}$ & $43,0 \mathrm{aB}$ & $8.5 \mathrm{aD}$ & $44,0 \mathrm{aC}$ & $75,5 \mathrm{aA}$ & $56,5 \mathrm{aB}$ & $42,63 \mathrm{a}$ \\
\hline Brisa IPA 12 & $0.0 \mathrm{bB}$ & $22,0 \mathrm{abA}$ & $30,5 \mathrm{bA}$ & $22,0 \mathrm{bA}$ & $1.5 \mathrm{aC}$ & $36,0 \mathrm{aA}$ & $41,0 \mathrm{bcA}$ & $18,0 \mathrm{cB}$ & $21,38 \mathrm{~b}$ \\
\hline Total dos substratos & $18,0 \mathrm{D}$ & $19,7 \mathrm{C}$ & $49,9 \mathrm{~A}$ & $33,8 \mathrm{~B}$ & $12,5 \mathrm{D}$ & $28,4 \mathrm{C}$ & $54,9 \mathrm{~A}$ & $36,6 \mathrm{~B}$ & \\
\hline $\mathrm{CV}(\%)=24,48$ & & & & & & & & & \\
\hline
\end{tabular}

$\mathrm{BT}=$ método do blotter test $\mathrm{AA}=$ meio ágar - água; $\mathrm{BDA}=$ meio batata - dextrose - ágar; $\mathrm{V} 8=$ suco de $\mathrm{V} 8-\mathrm{CaCO}_{3}-$ ágar. $\mathrm{Médias}$ seguidas de mesma letra minúscula na coluna e maiúscula na linha são estatisticamente iguais, de acordo com o teste de Tukey $(\mathrm{p} \leq 0,05)$. Dados transformados para Arco-seno raiz $((\mathrm{x}+1) / 100)$. A interação cultivar $\times$ substrato $\times$ temperatura foi significativa.

período, após desdobramento da bainha (a partir da umbela e com floretes fechados), até a formação de cápsula. Além disso, o A. niger pode ser transmitido de sementes de cebola infestadas para sementes e solo (KÖYCÜ; ÖZER, 1997). A maior incidência deste patógeno indica possíveis problemas de armazenamento das cultivares de cebola estudadas, já que este é influenciado por diversos fatores, dentre eles, temperatura e umidade relativa (CARVALHO; NAKAGAWA, 2000).

\section{Conclusões}

- O meio BDA foi o substrato que melhor proporcionou maior desenvolvimento fúngico, nos métodos estudados;

- No congelamento de sementes de cebola, BDA e V8 foram os substratos mais sensíveis para detecção de A. niger e A. flavus, respectivamente;

- Aspergillus niger foi o fungo mais frequentemente detectado em todos os tratamentos estudados; e 
- As cultivares IPA-11 e IPA-6 obtiveram altas percentagens de A. niger.

\section{Referências}

ALMEIDA, M. F.; REIS, E. M. Comparação da sensibilidade de métodos para a detecção de fungos patogênicos em sementes de aveia branca e preta no Rio Grande do Sul. Tropical Plant Pathology, v. 34, p. 265-269, 2009. http://dx.doi.org/10.1590/ S1982-56762009000400011

BOFF, P. et al. Estado sanitário de semente de cebola comercializada em Santa Catarina. Revista Brasileira de Sementes, v. 17, p. 165-170, 1995.

BRASIL. Ministério da Agricultura, Pecuária e Abastecimento. Manual de análise sanitária de sementes. Brasília: MAPA, 2009. 200 p.

CARVALHO, N. M.; NAKAGAWA, J. Sementes: ciências, tecnologia e produção. Jaboticabal: FUNEP, 2000. 588 p.

COUTINHO, W. M. et al. Uso da restrição hídrica na embebição ou retardamento da germinação de sementes de arroz e feijão submetidas ao teste de sanidade em meio ágar-água. Revista Brasileira de Sementes, v. 23, p. 127-135, 2001.

EL-NAGERABI, S. A. F.; ABDALLA, R. M. O. Note: Survey of seedborne fungi of sudanese cultivars of onion, with new records. Phytoparasitica, v. 32, p. 413-416, 2004. http://dx.doi.org/10.1007/ BF02979854

HILL, J. P. Twister. In: SCHWARTZ, H. F.; MOHAN, S. K. Compendium of onion and garlic diseases. St. Paul: APS Press, 2008. p. 26.

KLOKOÅAR-ŠMIT, Z. D. et al. Fusarium rot of onion and possible use of bioproduct. Matica Srpska Proceedings for Natural Science, n. 114, p. 135-148, 2008. http://dx.doi.org/10.2298/ ZMSPN0814135K

KÖYCÜ, N. D.; ÖZER, N. Determination of seedborne fungi in onion and their transmission to onion sets. Phytoparasitica, v. 25, n. 1, p. 25-31, 1997. http://dx.doi.org/10.1007/BF02981476
LORBEER, J. W.; RANSOM, V. E.; TUFFLEY, J. J. Nature and source of inoculum of Aspergillus niger causing the Aspergillus black mold disease of onions in New York. Ithaca: Cornell University, 2000. Results of the 2000 Agricultural Extension and Research Proyects Funded by the New York State IPM Program. Disponível em: <http://www.nysipm.cornell.edu/grantspgm/ projects/proj00/veg/lorbeer.asp>. Acesso em: 01 ago. 2011.

LUCCA FILHO, O. A. Metodologia dos testes de sanidade de sementes. In: SOAVE, J.; WETZEL, M. M. V. S. (Eds.). Patologia de sementes. Campinas: Fundação Cargill, 1987. p. 276-298.

MANNERUCCI, G. F. et al. Specie de Fusarium in seme di cipolla di produzione nazionale (Fusarium species in onion seed of Italian origin). Phytopathologia Mediterranea, v. 26, p. 156-64, 1987.

MASSOLA JUNIOR, N. S.; JESUS JUNIOR, W. C.; KIMATI, H. Doenças do alho e da cebola (Allium sativum L. e Allium cepa L.). In: KIMATI, H. et al. (Eds.). Manual de fitopatologia: doenças das plantas cultivadas. São Paulo: Agronômica Ceres, 2005. v. 2, p. 53-63.

MADDOX, D. A. Implications of new technologies for seed health testing and the worldwide movement of seed. Seed Science Research, v. 8, p. 277-284, 1998. http://dx.doi.org/10.1017/ S0960258500004177

MENTEN, J. O. M. Patógenos em sementes: detecção, danos e controle químico. São Paulo: Ciba Abro, 1995. 321 p

NASCIMENTO, W. M. O. et al. Qualidade sanitária e germinação de sementes de Pterogyne nitens Tull. (Leguminosae - Caesalpinioideae). Revista Brasileira de Sementes, v. 28, n. 1, p. 149-153, 2006. http://dx.doi.org/10.1590/ S0101-31222006000100021

NEERGAARD, P. Seed pathology. London: The Macmillan Press, 1983. v. 1, 839 p.

REIS, E. M. et al. Comparison of methods to detect leaf and head blighting fungi in small grain seeds. Summa Phytopathologica, v. 25, p. $364-367,1999$. 